\title{
Possible hot spots excited by the relativistic jets of Cygnus X-3
}

\author{
J. Martí1 ${ }^{1}$, D. Pérez-Ramírez ${ }^{1}$, J. L. Garrido ${ }^{1}$, P. Luque-Escamilla ${ }^{2}$, and J. M. Paredes ${ }^{3}$ \\ ${ }^{1}$ Departamento de Física, EPS, Universidad de Jaén, Campus Las Lagunillas s/n, Edif. A3, 23071 Jaén, Spain \\ e-mail: [jmarti;dperez;jlg]@ujaen.es \\ 2 Dpto. de Ing. Mecánica y Minera, EPS, Universidad de Jaén, Campus Las Lagunillas s/n, Edif. A3, 23071 Jaén, Spain \\ e-mail: peter@ujaen.es \\ 3 Departament d'Astronomia i Meteorologia, Universitat de Barcelona, Av. Diagonal 647, 08028 Barcelona, Spain \\ e-mail: jmparedes@ub.edu
}

Received 10 March 2005 / Accepted 27 April 2005

\begin{abstract}
We present the results of a deep search for associated radio features in the vicinity of the microquasar Cygnus X-3. The motivation behind is to find out evidence for interaction between its relativistic jets and the surrounding interstellar medium, which could eventually allow us to perform calorimetry of the total energy released by this microquasar during its flaring lifetime. Remarkably, two radio sources with mJy emission level at centimeter wavelengths have been detected in excellent alignment with the position angle of the inner radio jets. We propose that these objects could be the hot spots where the relativitic outflow collides with the ambient gas in analogy with Fanaroff-Riley II radio galaxies. These candidate hot spots are within a few arc-minutes of Cygnus X-3 and, if physically related, the full linear extent of the jet would reach tens of parsecs. We discuss here the evidence currently available to support this hypothesis based on both archival data and our own observations.
\end{abstract}

Key words. stars: individual: Cygnus X-3 - radio continuum: stars - X-rays: binaries

\section{Introduction}

Cygnus X-3 is a well known microquasar in the Galaxy, i.e., a X-ray binary producing bipolar relativistic jets. The neutron star or black hole nature of the compact object in the binary system is still a matter of discussion, but a consensus has been reached about the normal companion being a WN Wolf-Rayet star (Fender et al. 1999). The source is seen through an absorption of at least $A_{\mathrm{V}}=10 \mathrm{mag}$ and possibly higher. The relativistic outflow of this microquasar has been observed at both arc-second and sub-arcsecond scales, with an ejection axis very close to the North-South direction (see e.g. Martí et al. 2001; Miller-Jones et al. 2004). The reader is also referred to these references for a basic description of the main properties of this system. Here, we focus our attention on the unsolved problem of finding evidence for the expected hot spot/double lobe structures at the jet terminal shocks. The absence of observational evidence for such terminal features is an open question and not only for Cygnus X-3 itself but in a general context as well. Galactic sources of relativistic jets or microquasars pump significant amounts of energy into the interstellar medium (ISM). How such relativistic flows affect the galactic ISM remains, unfortunately, difficult to observe and study. As an illustrative example, simple power estimates by Fender \& Pooley (2000) suggest that $\sim 10^{51}$ erg (i.e. comparable with a supernova explosion) are injected by the superluminal microquasar GRS $1915+105$ into its surroundings over its total ejecting life time. Only in few cases we do see evidence of interaction between the jets and its surroundings, as in the double lobes of the Galactic Center microquasars 1E 1740.7-2942 (Mirabel et al. 1992) and GRS 1758-258 (Rodríguez et al. 1992), or a clear deceleration against the ISM as in the remarkable case of XTE J1550-564 (Corbel et al. 2002). Recently, a possible extended bow shock with arc-shaped morphology has been also reported at radio wavelengths for the microquasar Cygnus X-1 (Gallo et al. 2005).

The known population of galactic microquasars has grown significantly in recent times up to a total of at least 15 systems currently catalogued (Paredes \& Martí 2003). Nevertheless, the observational evidence of interaction between their relativistic jets and the ambient gas continues being the exception rather than the rule. Indeed, one would expect to see several examples of hot spot/double lobe structures at the jet terminal shocks in a process similar to extragalactic Fanaroff-Riley type II (FRII) sources (Fanaroff \& Riley 1974). In contrast, most relativistic jets in the Galaxy fade out before they significantly decelerate when their ram pressure equals that of the ISM.

This situation is indeed puzzling specially when comparing these relativistic jet sources with the thermal jets from young stellar objects (YSOs). Jets from YSOs are orders of magnitude less powerful than those of microquasars, but yet they are able to create a wide variety of bow shock structures. These 
are the well known Herbig-Haro objects whose latest catalog contains a few hundreds of entries (Reipurth 1999). Why such differences in number exist? Are YSOs bow shocks easier to observe only because they decelerate in a denser medium such as a molecular cloud with particle densities $\sim 10^{4} \mathrm{~cm}^{-3}$ ? Or is there an additional observational bias? Heinz (2002) has argued that microquasars are typically located in lower density environments, in a dynamical sense, than their AGN relatives. Tenuous media with densities well below the canonical $\sim 1 \mathrm{~cm}^{-3}$ would certainly imply the absence of interaction signatures, but additional work is needed to test whether this holds for most systems.

Early searches for large-scale structures connected with GRS 1915+105 revealed the existence of two intriguing IRAS sources whose radio emission is mostly of thermal freefree nature. They are symmetrically located with respect to GRS $1915+105$ and perfectly aligned with the position angle of its relativistic jets (Rodríguez \& Mirabel 1998; Chaty et al. 2001). A recent revision of their observational properties by Kaiser et al. (2004) concludes that it is perfectly conceivable, both energetically and dynamically, that the two IRAS sources are the jet impact regions on the ISM at a distance of tens of parsecs from their exciting source. Shock waves and turbulent plasma motion in the terminal lobes would naturally lead to synchrotron non-thermal emission explaining the unusual non-thermal feature in a otherwise normal HII region. Their conclusions are based on applying a scaled down model for large-scale structure of jets in extragalactic radio sources (Kaiser \& Alexander 1997). The average energy transport rate needed to sustain the proposed double structure is relatively low $\left(\sim 10^{36} \mathrm{erg} \mathrm{s}^{-1}\right)$, with an estimated age of $\sim 1 \mathrm{Myr}$. If the IRAS sources association with GRS $1915+105$ is confirmed, that would place this microquasar at a much lower distance than usually assumed.

In the case of the microquasar Cygnus X-3, a similar pair of symmetric radio sources aligned with the jets position angle was proposed by Martí et al. (2000). However, none of them displayed possible non-thermal filaments pointing towards the central core of Cygnus X-3 as in the GRS 1915+105 case. Moreover, their separation was exceedingly large $(\sim 100 \mathrm{pc})$ for a physical connection to be claimed without further evidence. All these facts induced us to carefully look for fainter and closer radio sources, not previously noticed, which could be associated with the missing large scale radio lobes. In this paper, we report the results of this search showing that Cygnus X-3 is likely another microquasar system where large-scale deceleration/interaction effects of the jets can be observed.

\section{Discovery of Cygnus X-3 hot spot candidates}

With the idea of searching for faint radio emission around Cygnus X-3 we have reanalyzed different observations retrieved from the archive of the Very Large Array (VLA) of NRAO in New Mexico (USA). The results reported here come mainly from three different VLA projects, namely those with identification codes AM551, AS483 and AM669. All of them were reduced using the AIPS package of NRAO applying standard procedures for calibration of interferometer data. All projects used 3C 286 as the amplitude calibrator and 2007+404 as the phase calibrator, at an angular distance of 4.7 from Cygnus X-3.

The first VLA project AM551 was conducted in 1997 by Martí et al. (2000) with the array in B configuration at the $6 \mathrm{~cm}$ wavelength being, in principle, sensitive to angular scales up to $\sim 30^{\prime \prime}$. Its main result was the detection of transient arcsecond radio jets of Cygnus X-3. Our hope when reanalyzing AM551 was that its deep integration time $(\sim 8 \mathrm{~h})$ could eventually reveal additional features fainter and/or further away from the microquasar core than those originally discovered. At the time of observations, Cygnus X-3 was in a "quiescent" state $\left(S_{6 \mathrm{~cm}} \sim 100 \mathrm{mJy}\right)$ and its variable radio core was subtracted with a similar approach as described by Martí et al. (2000) in order to remove artifacts induced by the microquasar variability during the CLEAN deconvolution process.

The resulting image is shown in the left panel of Fig. 1. Here, two faint radio sources are clearly detected at angular distances of 7.07 and 4.36 from Cygnus X-3 and roughly in the North-South direction. Interestingly, they are in almost perfect alignement with Cygnus X-3 along a position angle of $1.8 \pm 0.1$. This value agrees well with the position angle of the Cygnus X-3 inner arc-second radio jets, measured to be $2.0 \pm 0.4$ by Martí et al. (2001) Moreover, the asymmetry between the separation of the northern and southern sources is reminiscent of the asymmetry also existing between the northern and southern inner jet components (see the central right panel of Fig. 1). Such asymmetry could be accounted for if the northern and southern jets decelerate in regions of moderately different ISM density.

The observational properties of these newly discovered radio sources are summarized in Table 1. Wide field and zoomed contour maps are also presented in the different panels of Fig. 1. At their distances from the phase center, the new sources suffer from significant bandwidth smearing giving them an elongated shape poiting towards Cygnus X-3, specially in the northern case. Such instrumental limitation makes very difficult to assess which is their intrinsic morphology and this is the reason why angular diameters are not included in Table 1. Nevertheless, the possibility that the sources are slightly extended cannot be ruled out based on present data. Indeed the slightly curved shape of the northern object is not strictly consistent with a pure radial smearing of a compact source. A crude estimate of its transversal angular size can be obtained by taking the deconvolved minor axis of an elliptical Gaussian fit using the AIPS task IMFIT. This size is about 1". 6 and we will later adopt an aspect ratio of 1 for discussion purposes.

From source counts (see Rodríguez et al. 1989) and simple geometric arguments, we estimate that the probability of randomly find two background radio sources brighter than $1 \mathrm{mJy}$, aligned within $\sim 1^{\circ}$ of the jet position angle and closer than 7'.0 from Cygnus X-3, is of order $\sim 10^{-5}$ percent. Indeed, no other pair of radio sources but these two appear so well aligned in the field.

In order to estimate the source's spectral index $\alpha$ (defined as $S_{v} \propto v^{\alpha}$, where $S_{v}$ is the radio flux density at a given frequency $v$ ), and hence constraining the emission mechanism, we searched the VLA archives for suitable observations at a 


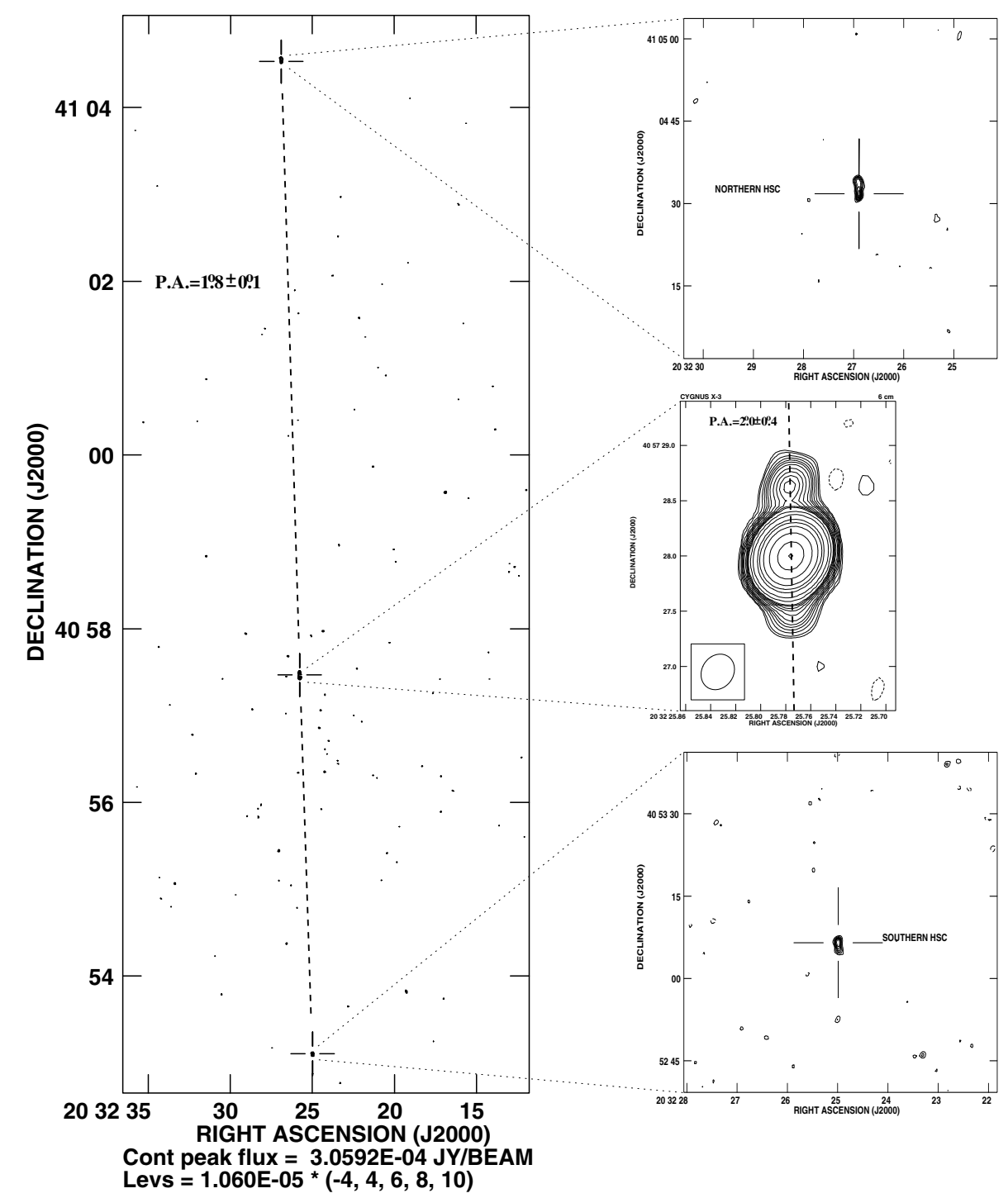

Fig. 1. Left: natural weight map of the Cygnus X-3 field from a re-analysis of VLA project AM551. The original observation was conducted on 4 May 1997 at the $6 \mathrm{~cm}$ wavelength in B configuration. The map covers almost the totality of the antenna primary beam and remarkably shows the existence of two radio sources perfectly aligned with the position angle of the Cygnus X-3 inner radio jets. These are the only objects in the field with peak flux density above four times the rms noise of $10.6 \mu \mathrm{Jy}$ beam $^{-1}$, in addition to Cygnus X-3 itself. We interpret them as the possible hot spots where a larger scale flow terminates. The clean components have been restored using an almost circular Gaussian beam of 1".5. Right: the top and bottom panels are zoomed contour plots of the two hot spot candidates. Their morphology is difficult to judge because of severe bandwidth and time smearing at their distance from the phase center, although they could be slightly resolved. The central panel shows the Cygnus X-3 arc-second radio jets taken from Martí et al. (2001) for illustrative purposes and corresponding to a strong radio outburst in late 2000.

Table 1. Radio properties of possible hot spots associated with Cygnus X-3 derived from VLA observations.

\begin{tabular}{cccccc}
\hline \hline $\begin{array}{c}\text { Hot spot } \\
\text { candidate }\end{array}$ & $\begin{array}{c}\text { Position } \\
\alpha_{\mathrm{J} 2000.0}, \delta_{\mathrm{J} 2000.0}\end{array}$ & $\begin{array}{c}\text { Frequency } \\
(\mathrm{GHz})\end{array}$ & $\begin{array}{c}\text { Peak flux density* } \\
(\mathrm{mJy})\end{array}$ & $\begin{array}{c}\text { Integrated flux density* } \\
(\mathrm{mJy})\end{array}$ & $\begin{array}{c}\text { Spectral } \\
\text { index }\end{array}$ \\
\hline North & $20^{\mathrm{h}} 32^{\mathrm{m}} 26^{\mathrm{s}} 88 \pm 0.01$ & 1.425 & $1.63 \pm 0.13$ & $3.3 \pm 0.5$ & $-0.4 \pm 0.2$ \\
& $+41^{\circ} 04^{\prime} 32^{\prime \prime} 9 \pm 0.9$ & 4.860 & $1.25 \pm 0.11$ & $1.9 \pm 0.4$ & $\geq-0.7^{* *}$ \\
& & & & - \\
South & $20^{\mathrm{h}} 32^{\mathrm{m}} 24.97 \pm 0.02$ & 1.425 & $\leq 0.6$ & $0.45 \pm 0.09$ & \\
& $+40^{\circ} 53^{\prime} 05^{\prime \prime} \cdot 9 \pm 0.2$ & 4.860 & $0.27 \pm 0.04$ & & \\
\hline
\end{tabular}

* Corrected for primary beam decay using the AIPS task PBCOR but not for bandwidth smearing.

** Computed using the peak and $4 \sigma$ upper limit values corrected for bandwidth smearing. 
different wavelength. The VLA project AS483 conducted in year 1993 by A. Smale in B configuration was found to be appropriated for such purpose. A re-analysis of it provided a clear detection of the northern radio source, at the $20 \mathrm{~cm}$ wavelength, and an upper limit to the brightness of the southern one. The $20 \mathrm{~cm}$ results and the resulting spectral index information are also included in Table 1. Using the Smale data, the radio spectrum of the northern candidate is well described by $S_{v}=3.9 \pm$ $0.4 \mathrm{mJy}[v / \mathrm{GHz}]^{-0.4 \pm 0.2}$, which clearly suggests a non-thermal synchrotron emission mechanism. The spectral index of the southern source is also consistent with a similar negative value, i.e, typical of optically thin synchrotron radiation.

Taking together i) the almost perfect alignment with the jet's position angle; ii) the north/south asymmetry similar to that of the arc-second radio jets; and iii) the likely non-thermal emission of the two radio sources discovered, we propose them as serious candidates to be the impact sites of the Cygnus X-3 jets against the ISM. Hereafter, we will refer to them as the northern and southern hot spot candidates (HSCs).

\section{Setting upper limits on proper motion}

Following their discovery, the two HSCs were also detected when carefully inspecting very wide field VLA maps obtained from the AM669 data. This project was carried out during late 2000 with the array being in its most extended A configuration and published by Martí et al. (2001). The new AM669 maps (not shown here) also suffer from noticeable smearing like those of Fig. 1. However, both the northern and southern hot spot candidates were detected although with low signal-to-noise ratios of 5 and 4, respectively. Using the longer time baseline available, 7.6 yr between the AM669 and AS483 data, we can try to set an upper limit to the proper motion of the north HSC with respect to Cygnus X-3. The total projected length of the northern flow $L_{j} \sin \theta / D$ would be equivalent to 7!.07, where $\theta$ is the angle with the line of sight and $D$ the distance to the source. Between 1993 and 2000, the north HSC shifted its separation from Cygnus X-3 by less than about 0.7 . This value is comparable or smaller than one synthesized beam for any of the two observing epochs involved. Considering that smearing is also likely to affect our $6 \mathrm{~cm}$ astrometry at large distances from the phase center, we conservatively consider that no reliable proper motion can be claimed based on the present data. Therefore, a safe upper limit on the advance speed of the end of the possible jet can be expressed as $\dot{L}_{j} \leq 4400 \mathrm{~km} \mathrm{~s}^{-1} D_{10}(\sin \theta)^{-1}$ being $D_{10}$ the source distance in units of $10 \mathrm{kpc}$.

\section{Search for X-ray, optical and infrared counterparts}

In order to exclude the possible stellar or extragalactic nature for the HSCs, their accurate radio positions in Table 1 have been searched at X-ray, optical and infrared wavelengths.

\subsection{X-rays}

We have inspected the HSCs location in several X-ray mission archives, including ROSAT, XMM and CHANDRA. We only found a ROSAT PSPC image (0.76-2.04 KeV band) from which an upper limit count rate of 6.245 counts $\mathrm{s}^{-1}$ was inferred. Assuming a photon index $\Gamma=1.5$ and an absorption of $A_{\mathrm{V}}=10$ mag towards Cygnus $\mathrm{X}-3$, equivalent to $N_{\mathrm{H}}=1.8 \times$ $10^{22} \mathrm{~cm}^{-2}$ (Predehl \& Schmitt 1995), the corresponding unabsorbed X-ray flux limit is $225 \mu \mathrm{Jy}$.

\subsection{Optical observations}

In the optical domain, we observed the two positions with the $2.2 \mathrm{~m}$ telescope at the Centro Astronómico Hispano Alemán (CAHA) in Calar Alto (Spain). We obtained deep I-band images using the SITe-d15 CCD detector of the CAFOS instrument. Astrometry of the CCD frames was determined through twenty stars in the field whose positions were retrieved using the SkyCat tool of the European Southern Observatory. The plate solutions were established by means of the AIPS task XTRAN with a third order polinomial fit, whose residuals were typically of 0.5 .

Figure 2 shows the results of our CAHA observations and astrometry. Cygnus X-3 was clearly detected in the $I$-band with an estimated magnitude of about $I=21.3$, which is comparable to previous detections reported in the literature (Wagner et al. 1989). In contrast, no optical counterpart was detected at the likely hot spot location up to a limit magnitude of $I=22.4$.

\subsection{Infrared search}

The search for a counterpart at near-infrared wavelengths is strongly justified given the strong interstellar absorption in the Cygnus X-3 region. The 2 Micron All Sky Survey (2MASS) was also inspected at the position of the HSCs. A near-infrared counterpart was not found for none of them within 4 " of their radio position. The corresponding upper limit magnitudes are $17.8,16.8$ and 16.3 for the $J, H$ and $K$ s bands, respectively.

\section{Discussion}

The observed radio spectrum and infrared and X-ray upper limits are consistent with extrapolation of the same spectral index derived from 20 and $6 \mathrm{~cm}$ observations (see Fig. 3). In the previous sections, we have failed to identify an optical and nearinfrared counterpart with stellar appearance for the HSCs. Had they been background or foreground stars, or nearby AGNs, we would expect to detect some optical or infrared source with compact appearance unless absorption prevents us from doing so. In any case, the possibility that we are not dealing with stars or AGNs remains clearly open. Thus, we will proceed hereafter under the assumption that both HSCs are physically related to Cygnus X-3 although this point needs yet to be confirmed by further more sensitive observations.

During our discussion we will apply some of the equations used by Kaiser et al. (2004) when revising the possible large scale structure of GRS 1915+105. In their work, thermal 


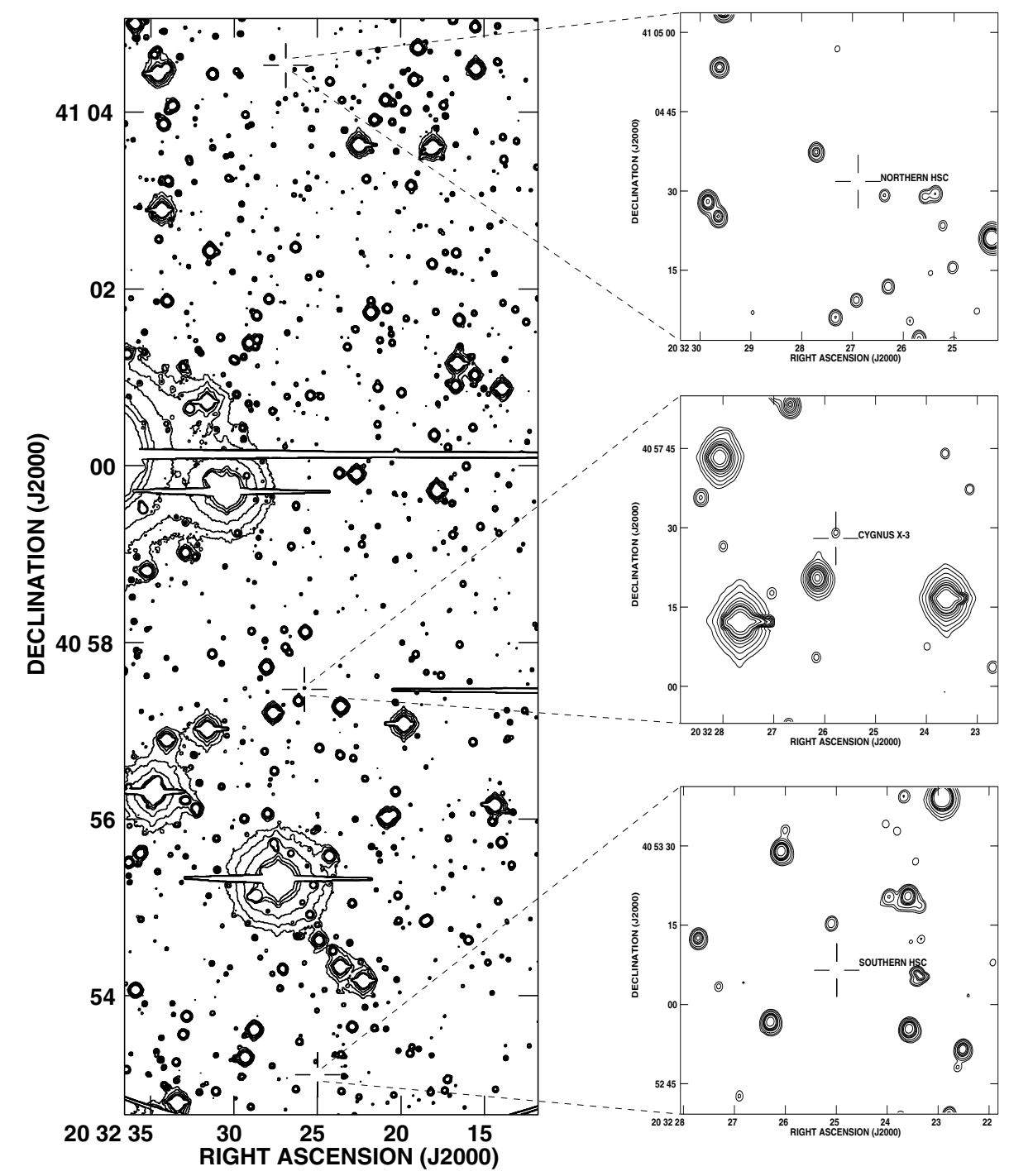

Fig. 2. CAHA $2.2 \mathrm{~m} \mathrm{I-band} \mathrm{image} \mathrm{taken} \mathrm{on} 2004$ July 20 with the CAFOS instrument. The crosses with a gap mark both the position of Cygnus X-3 and the two hot spot candidates. The right panels display an enlarged view of their respective locations.

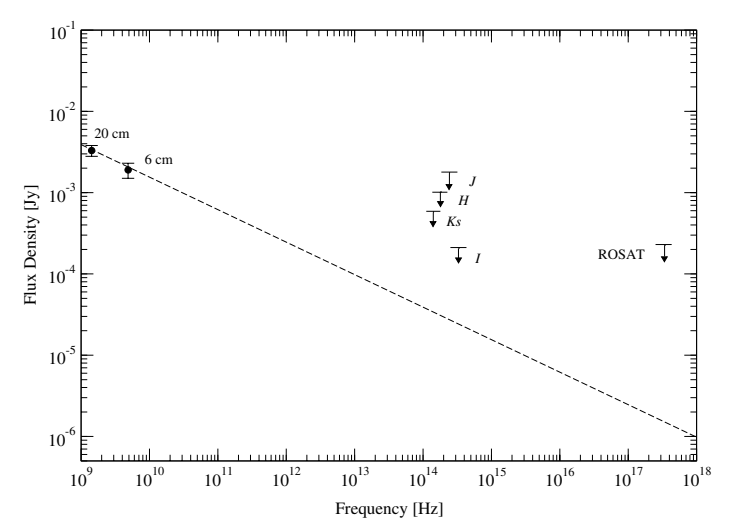

Fig. 3. Spectral energy distribution for the northern HSC expected at infrared and X-ray wavelengths extrapolating a synchrotron spectrum with the same power law index as derived from radio observations (dashed line). The filled triangles represent upper limits for the optical (CAHA), near-infrared (2MASS) and X-ray (ROSAT) emission after reddening correction assuming $A_{\mathrm{V}}=10 \mathrm{mag}$. Higher absorption values, certainly not excluded for this source, would just rise the upper limits plotted here not affecting the conclusions of our paper. bremsstrahlung radio emission is detected from the shock compressed ISM (i.e. the extended IRAS sources). Unfortunately, our application of their formalism will necessarily be limited because we did not find evidence of such thermal emission downstream of the HSC. The $4 \sigma$ upper limits at $6 \mathrm{~cm}$ are 0.6 and $0.1 \mathrm{mJy} \mathrm{beam}^{-1}$ for the northern and southern one, respectively. The possibility that such emission exists is, however, not ruled out by our present VLA observations due to lack of sensitivity so far away from the phase center and the blindness of the VLA B configuration array to angular scales larger than half arc-minute.

It is instructive to assess here the possibility for Cygnus X-3 to create a pair of hot spots at tens of parsec distance through estimating its average energy injection rate. Martí et al. (1992) have modelled the strong radio outbursts of Cygnus X-3 in terms of laterally expanding twin jets with particle injection at the jet basis. According to this model, a flaring event with cm peak flux density of 10-20 Jy involves a total energy of $\sim 10^{44}$ erg in relativistic particles. Based on several years of radio monitoring of Cygnus X-3 with the Green Bank 
interferometer (Waltman et al. 1994), two of such events occur typically per year. Thus we find that $\sim 10^{37} \mathrm{erg} \mathrm{s}^{-1}$ can be adopted as a conceivable estimate. A similar value is obtained if one considers the quiescent (low-level) radio flares of Cygnus X-3, whose energy content is about two orders of magnitude lower and evolve within time scales of few hours. Remarkably, this energy injection rate is in close accordance with the corresponding value estimated by Kaiser et al. (2004) for the superluminal microquasar GRS $1915+105$. Such agreement strongly suggests that both microquasars could sustain similar double lobe/hot spot features when their relativistic ejecta interact with the ISM.

The non-thermal spectrum of the Cygnus X-3 HSCs agrees with the expected synchrotron emission mechanism, suggesting the existence of relativistic particles with a magnetic field. Assuming standard equipartition arguments and a $10 \mathrm{kpc}$ distance, the minimum energy requirements and associated physical parameters can be estimated by using the observed flux density and angular size (Longair 1994). Provided that the magnetic field covers the entire radio source volume and electrons and protons share equal energies, this implies a minimum energy of $\sim 8 \times 10^{43} \mathrm{erg}$. The corresponding magnetic field is then $3 \times 10^{-4} \mathrm{G}$ and the total pressure $1.8 \times 10^{-9} \mathrm{erg} \mathrm{cm}^{-3}$. These values are within a factor of a few as compared to the non-thermal feature next to the southern lobe candidate of GRS 1915+105 (Kaiser et al. 2004).

The observed angular separation between Cygnus X-3 and its possible hot spots transform into a linear size of 20 and $13 \mathrm{pc}$ times $D_{10}(\sin \theta)^{-1}$ for the northern and southern candidates, respectively. These values are roughly a factor of three smaller than those of GRS $1915+105$. They are not expected to significatively change even in time scales of many years. Indeed, for a strong shock in monoatomic gas the advance velocity of the end of the jet can be expressed as

$\dot{L}_{j} \sim \sqrt{\frac{16 k_{\mathrm{B}} T}{3 m_{\mathrm{H}}}}$,

where $k_{\mathrm{B}}$ is the Boltzmann constant, $m_{\mathrm{H}}$ the proton mass and $T$ the temperature of the shocked gas (Kaiser et al. 2004). Assuming $T \gtrsim 10^{4} \mathrm{~K}$ for the post-shock temperature of the bremsstrahlung emitting bow-shock (yet undetected for Cygnus X-3), one expects a lobe advance velocity of $\gtrsim 20 \mathrm{~km} \mathrm{~s}^{-1}$. This value is well consistent with the proper motion upper limit derived in a previous section from observations with a time baseline of several years. Using again the Kaiser \& Alexander (1997) formalism for a supersonically expanding lobe into a constant density medium, the corresponding age of the jet is given by the following self-similar solution

$t=\frac{3}{5} \frac{L_{j}}{\dot{L_{j}}}$,

which yields an age of $\lesssim 0.6$ Myr. This upper limit satisfactorily agrees with the expected lifetime of a Wolf-Rayet star, which is determined by the nuclear burning time of its helium core. For a massive binary system, the core mass is expected to be $M_{1} \simeq 8 M_{\odot}$, while the nuclear burning time of helium depends as (Lipunov 1992)

$t_{\mathrm{He}} \simeq 3 \times 10^{6} \mathrm{yr}\left[M_{1} / M_{\odot}\right]^{-0.7}$.
This yields $t_{\mathrm{He}} \simeq 0.7 \mathrm{Myr}$ which would be satisfactorily agree with the estimated jet age.

\section{Conclusions}

Based on a deep VLA map at the $6 \mathrm{~cm}$ wavelength, we have discovered two radio sources in the vicinity of Cygnus X-3 which we propose to be the hot spots of its relativistic jets. Assuming this statement to be correct, the total extent of the jet flow would be measured in tens of parsecs. The proposed physical association is based on the following evidences:

1. The line joining the two HSCs agrees within one degree with the position angle of the inner arcsecond and subarcsecond jets.

2. Their spectral index is consistent with a non-thermal emission mechanism, which would naturally be expected from synchrotron radiation in the jet bow-shocks.

3. We find no evidence for a point-like optical and near infrared counterpart, as one would expect if the radio sources were stellar or AGN in nature.

4. The physical parameters of the HSCs (magnetic field, pressure, energy density, advance velocity, etc.) are comparable to those of the proposed hot spots in GRS 1915+105 The possible existence of similar structures in both microquasars should not come with surprise since their estimated energy injection rate into the ISM is also very similar $\left(\sim 10^{37} \mathrm{erg} \mathrm{s}^{-1}\right)$.

5. For Cygnus X-3, the kinematical age of the proposed hot spot complex would be consistent with the expected lifetime of the Wolft-Rayet stage in the binary system.

If the hot spot nature is confirmed by further observations, the analogy between microquasars and their extragalactic relatives would be extended beyond the accretion/ejection point of view, and include the interaction of their relativistic flows with the surrounding medium, either interstellar or intergalactic. We can even especulate that a dichotomy, similar to that of Fanaroff-Ryle type I and II radiogalaxies, could also exist in the microquasar domain.

Acknowledgements. The authors acknowledge support by DGI of the Ministerio de Educación y Ciencia (Spain) under grants AYA2004-07171-C02-02 and AYA2004-07171-C02-01, FEDER funds and Plan Andaluz de Investigación of Junta de Andalucía as research group FQM322. The National Radio Astronomy Observatory is a facility of the National Science Foundation operated under cooperative agreement by Associated Universities, Inc. in the USA. This paper is also based on observations collected at the Centro Astronómico Hispano Alemán (CAHA) at Calar Alto, operated jointly by the Max-Planck Institut für Astronomie and the Instituto de Astrofísica de Andalucía (CSIC). This research has made use of the SIMBAD database, operated at CDS, Strasbourg, France. We also used the data products from the Two Micron All Sky Survey, which is a joint project of the University of Massachusetts and the Infrared Processing and Analysis Center/California Institute of Technology, funded by the National Aeronautics and Space Administration and the National Science Foundation of USA. The research of DPR has been supported by the Education Council of Junta de Andalucía (Spain). 


\section{References}

Chaty, S., Rodríguez, L. F., Mirabel, I. F., et al. 2001, A\&A, 366, 1035 Corbel, S., Fender, R. P., Tzioumis, A. K., et al. 2002, Science, 5591, 196

Fanaroff, B. L., \& Riley, J. M. 1974, MNRAS, 167, 31

Fender, R. P., Hanson, M. M., \& Pooley, G. G. 1999, MNRAS, 308, 473

Fender, R. P., \& Pooley, G. G. 2000, MNRAS, 318, L1

Gallo, E., Fender, R. P., \& Kaiser, C. R. 2005 [arXiv: astro-ph/0501374]

Heinz, S. 2002, A\&A, 388, L40

Kaiser, C. R., \& Alexander, P. 1997, MNRAS, 286, 215

Kaiser, C. R., Gunn, K. F., Brocksopp, C., \& Sokoloski, J. L. 2004, ApJ, 612, 332

Lipunov, V. M. 1992, Astrophysics of Neutron Stars (Springer Verlag)

Longair, M. S. 1994, High Energy Astrophysics (Cambridge University Press)

Martí, J., Paredes, J. M., \& Estalella, R. 1992, A\&A, 258, 309
Martí, J., Paredes, J. M., \& Peracaula, M. 2000, ApJ, 545, 939

Martí, J., Paredes, J. M., \& Peracaula, M. 2001, A\&A, 375, 476

Miller-Jones, J. C. A., Blundell, K. M., Rupen, M. P., et al. 2004, ApJ, 600,368

Mirabel, I. F., Rodríguez, L. F., Cordier, B., Paul, J., \& Lebrun, F. 1992, Nature, 358, 215

Paredes, J. M., \& Martí, J. 2003, Contributions to Science, 2(3), 303, http://www.cat-science.com/

Predehl, P., \& Schmitt, J. H. M. M. 1995, A\&A, 293, 889

Reipurth, B. 1999, A general catalogue of Herbig-Haro objects, 2nd Ed., http://casa. colorado.edu/hhcat

Rodríguez, L. F., Myers, P. C., Cruz-González, I., \& Terebey, S. 1989, ApJ, 347, 461

Rodríguez, L. F., Mirabel, I. F., \& Martí, J. 1992, ApJ, L15

Rodríguez, L. F., \& Mirabel, I. F. 1998, A\&A, 340, L47

Wagner, R. M., Kreidl, P. J., Bus, S. J., \& Williams, W. 1989, ApJ, 346, 971

Waltman, E. B., Fiedler, R. L., Johnston, K. J., \& Ghigo, F. D. 1994, AJ, 108, 179 